\title{
Commissioning the ATLAS Liquid Argon Calorimeter Phase-I Upgrade
}

\author{
Ellis Kay, on behalf of the ATLAS Liquid Argon Calorimeter Group \\ Department of Physics and Astronomy, University of Victoria, Victoria, BC, \\ V8W 2Y2. Canada \\ E-mail: ellis.kay@cern.ch
}

\begin{abstract}
Liquid argon (LAr) sampling calorimeters are employed by ATLAS for all electromagnetic calorimetry in the pseudo-rapidity region $|\eta|<3.2$, and for hadronic and forward calorimetry in the region from $|\eta|=1.5$ to $|\eta|=4.9$. In the first LHC run (Run-1), a total luminosity of around $26 \mathrm{fb}^{-1}$ was collected at centre-of-mass energies of 7-8 TeV. After detector consolidation during a long shut-down, Run-2 started in 2015 and around $150 \mathrm{fb}^{-1}$ of data at a centre-of-mass energy of $13 \mathrm{TeV}$ was recorded. With the end of Run-2 in 2018, a multi-year shutdown for the Phase-I detector upgrades began. As part of the Phase-I upgrade, new trigger readout electronics of the ATLAS LAr Calorimeter have been developed. Installation be-gan at the start of the LHC shut down in 2019 and is expected to be completed in 2020. A commissioning cam-paign is underway in order to realise the capabilities of the new, higher granularity and higher precision level-1 trigger hardware in Run-3 data taking. This contribution will give an overview of the new trigger readout system and the ongoing commissioning and installation efforts.
\end{abstract}

I. The ATLAS Liquid Argon Calorimeter

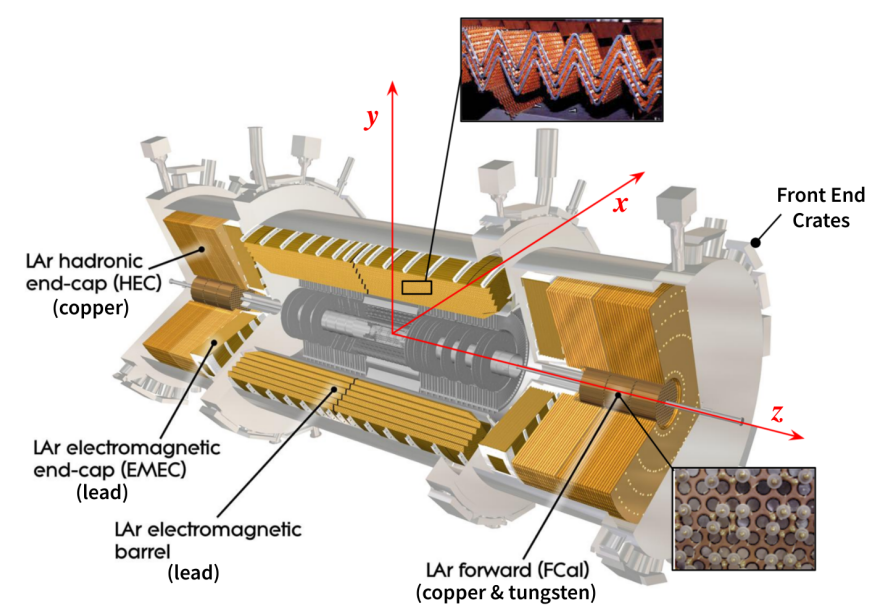

Figure 1: The ATLAS liquid-argon calorimeter system [1]. The different sections of the subdetector are labelled, along with their respective absorber materials. Photographs of the different structures of the forward calorimeters (FCal) and electromagnetic barrel (EMB) calorimeters are overlaid [2].

The ATLAS LAr calorimeter [3] is a sampling calorime- ter with full coverage in azimuthal angle日 1 . It uses liquid argon as an active medium, with copper electrodes and various absorber materials (see Figure 1). Incident particles shower in the absorber material and subsequently ionise the LAr. An electric field applied across the LAr gap causes the ionisation electrons to drift across it, leading to a triangular pulse which is collected by the electrodes (see Figure 2). The subdetector is housed in one 'barrel' and two 'endcap' cryostats kept at $-184^{\circ} \mathrm{C}$. A high granularity electromagnetic (EM) calorimeter with an accordion geometry covers the barrel (EMB) region of $|\eta|<1.475$ and the end-cap (EMEC) region of $1.375<|\eta|<3.2$. Two parallel-plate hadronic endcap (HEC) calorimeters cover the region $1.5<|\eta|<3.2$, while forward calorimeters (FCal), which use cylindrical electrodes running parallel to the beam axis within a honeycomb structure of metal, cover the region $3.1<|\eta|<3.9$. Each section of the calorimeter is segmented in depth in up to three layers, referred to as front, middle and back. For $|\eta|<1.8$, an additional 'presampler' layer provides measurements of energy lost upstream of the subdetector. The LAr calorimeter, with each of these technologies mirrored in the $\mathrm{A}$ and $\mathrm{C}$ sides of the detector, consists of 8 subsystems, referred to as 'partitions', namely EMBA, EMBC, EMECA, EMECC, HECA, HECC, FCalA and FCalC.

\section{Readout Electronics}

The ionisation signals from the 182486 LAr readout channels leave the cryostats via 114 feedthroughs. Each feedthrough is connected to one of the Half-Front-EndCrates (HFECs), which sit radially around the detector and house several kinds of electronic boards connected to a baseplane. Front-End Boards (FEBs) [5] are responsible for receiving the analogue signals from up to 128 channels and sampling and digitising them before transmitting them, via optical links, to the back-end electronics which reside in an underground service cavern separate from the

\footnotetext{
${ }^{1}$ ATLAS uses a right-handed coordinate system with its origin at the nominal interaction point (IP) in the centre of the detector and the $z$-axis along the beam pipe. The $x$-axis points from the IP to the centre of the LHC ring, and the $y$ axis points upward. Cylindrical coordinates $(r, \phi)$ are used in the transverse plane, with $\phi$ being the azimuthal angle around the $z$-axis. The pseudorapidity is defined in terms of the polar angle $\theta$ as $\eta=-\ln \tan (\theta / 2)$. In ATLAS, the ends of the detector with positive and negative $z$ coordinates are referred to as 'A-side' and 'C-side', respectively.
} 


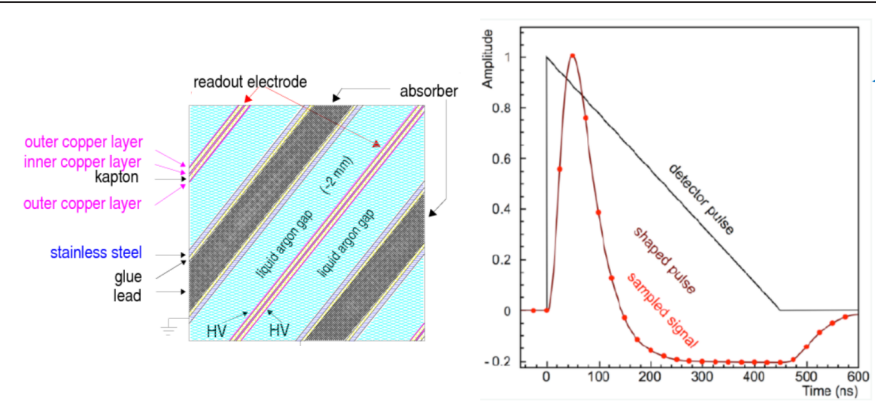

Figure 2: Diagrams of the structure of a sampling layer of the ATLAS EM calorimeter (left) and a pulse shape read out from the detector before and after shaping and sampling (right) [4].

experiment. The signal from each channel is split into one of three gains (low, medium and high) and, for each gain, the triangular ionisation pulse is shaped with a bipolar $\left(\mathrm{CR}-(\mathrm{RC})^{2}\right)$ analogue filter. The shaped signals are sampled at the LHC bunch-crossing frequency of $40 \mathrm{MHz}$ and the samples are stored in a Switched Capacitor Array (SCA) analogue memory buffer while awaiting a Level-1 (L1) trigger accept. In addition to reading out the individual calorimeter channels, the FEB performs the first stages of summing in preparation of analogue sums which are used by the L1 trigger system [6] to inform L1 accepts. Each of the shaper chips in the FEB outputs a partial sum of input channels, which are routed to two mezzanine boards known as Layer Sum Boards (LSBs) which perform further summing, depending on the number of layers in the relevant detector region. These sums are sent through the HFEC's baseplane to Tower Builder Boards (TBBs) or Tower Driver Boards (TDBs), which sum data from calorimeter cells in all layers in so-called Trigger Towers (TTs), which correspond to slices of the detector of approximately $\Delta \eta \times \Delta \phi=0.1 \times 0.1$. The HFECs also contain calibration boards, which are used to inject known triangular pulses into the cryostat in order to simulate the LAr ionisation signal and calibrate the electronics.

\section{Phase-I Upgrade}
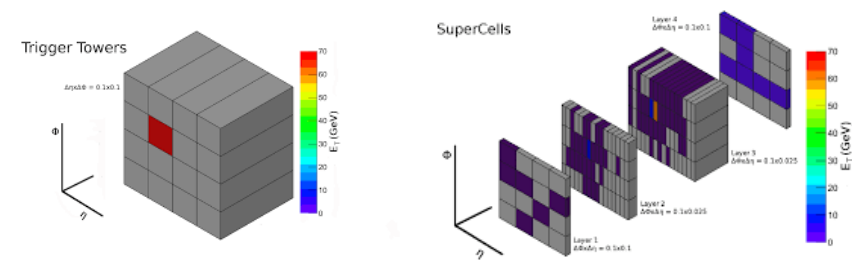

Figure 3: Diagrams showing the energy deposited by an electron with $E_{T}=70 \mathrm{GeV}$ as seen by a trigger tower (left) and by the equivalent $\mathrm{SCs}$ for a typical region in the EM calorimeter [7].

The instantaneous luminosity and pileup will increase for Run-3 of the LHC, yet the current ATLAS trigger system does not allow for a proportionate increase of the L1 trigger rate. In order to compensate for this while maintaining similar triggering performance, an improvement of the trigger system itself is required. Upgraded L1 algorithms will have better discrimination between photons, electrons, taus and jets, and new Feature EXtractor (FEX) modules will use topological shower shape variables to discriminate between these different objects [8]. Such improvements require higher granularity information from the LAr calorimeter than what is provided in the Run-2 trigger readout path. Currently, signals from cells in different calorimeter sampling layers are grouped into $\sim 5400$ trigger towers, as described in the previous section. As a result of this summing, information about the development of shower shapes is lost, and cannot be used as a discriminating variable in the trigger decision. In the Phase-I upgrade, cells will instead be grouped into 34000 'Super Cells' (SCs), which provide up to fourlayer information and a tenfold increase in granularity. Figure 3 shows a depiction of an electron energy deposit as seen through a TT compared to the same deposit measured by SCs for a typical EM $\eta, \phi$ slice. In this case, the SCs provide information for $\Delta \eta \times \Delta \phi=0.1 \times 0.1$ for the presampler and back layers and $\Delta \eta \times \Delta \phi=0.025 \times 0.1$ for the front and middle layers. Figure 4 shows the expected improvement in performance from the PhaseI LAr upgrade.
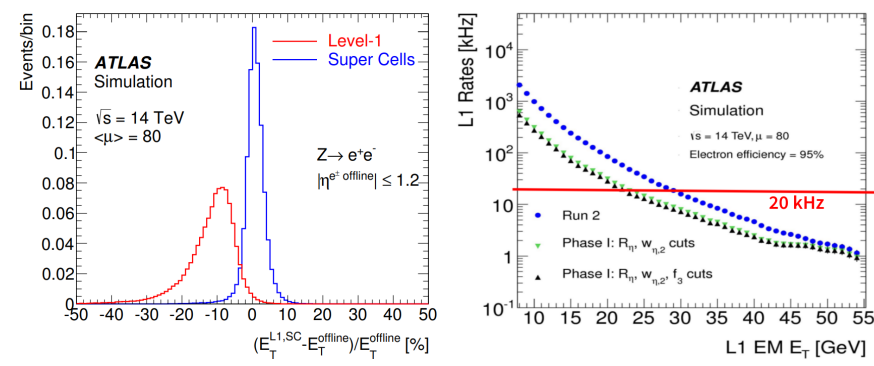

Figure 4: Expected performance of the Phase-I trigger upgrade [7]. In the plot on the left, the percent difference between the $E_{T}$ of electrons reconstructed offline $\left(E_{T}^{\text {offline }}\right)$ and using the Run-2 L1 is shown in red, while the difference between this offline reconstruction and the energy from SCs is shown in blue, where the $E_{T}$ has been simulated using $Z \rightarrow e^{+} e^{-}$events in the EMB calorimeter. The plot on the right shows expected L1 trigger rates for $\mathcal{L}=3 \times 10^{34} \mathrm{~cm}^{-2} \mathrm{~s}^{-1}$ as a function of the EM $E_{T}$ threshold for the case of the Run-2 trigger (blue) and the Phase-I trigger scheme with different selections of shower shape variables (black and green). A red line indicates the $\mathrm{L} 1$ limit of $20 \mathrm{kHz}$ for electromagnetic objects, which will remain in Run-3.

In order to transmit this higher granularity information from SCs to the L1 Calorimeter (L1Calo) system, the LAr front-end and back-end electronics must be upgraded. Figure 5 shows a schematic of the readout electronics, with these new changes shown in red. The most notable change to the front-end is the introduction of 124 new LAr Trigger Digitiser Boards (LTDBs), which serve to digitise the analogue signal of supercells, processing up to 320 SC signals each. There are seven different 'flavours' of these boards depending on their location, with one flavour for the barrel and six for the end-caps. In order to accommodate this new addition, the baseplanes in each FEC must be replaced with new ones, which have the necessary number of slots. These new baseplanes also deal with changes to the signal path which are introduced in this upgrade, including the routing of legacy trigger tower sums for some layers through the LTDB to the TBB, and 


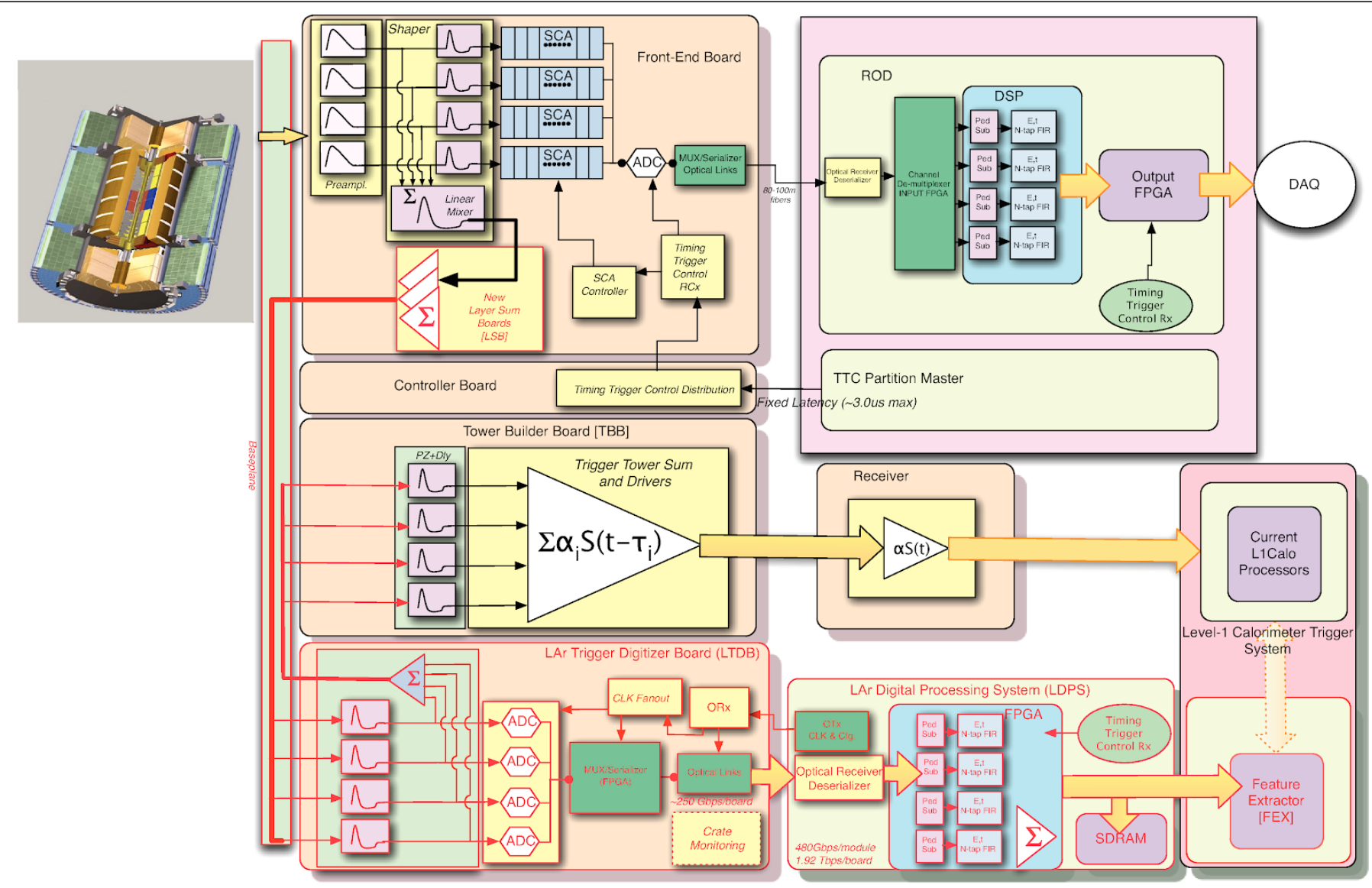

Figure 5: Schematic of the Phase-I upgrade architecture [7]. The new components are indicated by the red outlines and arrows. The main readout path (from FEBs to DAQ) and legacy trigger path (from FEBs to L1 via TBB) are also shown.

the routing of SC sums through the LSBs to the LTDB. The latter path also requires an exchange of the LSBs on all FEBs. The reception of this digital SC data and its transmission to the new FEXes is handled by new backend hardware, collectively referred to as the LAr Digital Processing System (LDPS). This system, connected to the LTDBs through a total of 7000 optical fibres, serves to receive $\mathrm{ADC}$ data and align timing, compute $E_{T}$ for individual SCs in each bunch crossing, sum $E_{T}$ for SCs over defined areas of the detector and send the result to the L1 trigger modules. The LDPS consists of advanced mezzanine cards, named LAr Trigger prOcessing MEzzanines (LATOMEs), and Advanced-TCA (ATCA) LAr Carrier (LArC) blades with Rear Transition Module (RTM) cards and Intelligent Platform Management Controller (IPMC) mezzanine cards [9]. Each set of LArC and four LATOMEs is referred to as a LAr Digital Processing Blade (LDPB). In total, the LDPS consists of 116 LATOMEs and 30 LArCs distributed across three ATCA shelves.

The main readout path, as described in section II], remains unchanged by this upgrade, though data must pass through the new baseplanes and refurbished FEBs. The legacy trigger path will be maintained during Run-3, running concurrently with the new digital trigger, and should also be unchanged, though trigger tower sums for some layers will be routed through the new LTDBs.

\section{Installation Status}

Over the course of the ongoing Long Shutdown (LS2) of the LHC in preparation for Run-3, the installation of the new front-end and back-end LAr electronics has been progressing. The removal and replacement of all baseplanes on the detector has been completed. This required, as a first step, the removal of all boards in each HFEC; a challenging tasks in a restricted space. In order to exchange the LSB on each FEB, every one of these boards needed to be removed from the cavern and refurbished. This has been achieved for all FEBs on the detector. Simultaneously, the cooling plates which surround each board were replaced with newly manufactured ones, as well as the ageing hoses that supply cold water to each board.

The installation of LTDBs continues at the time of this publication. All LTDBs were manufactured, tested and delivered to CERN, with just six barrel (EMBC) and four end-cap (EMECC) boards awaiting installation due to a lack of access to the relevant regions of the detector.

The back-end installation of LDPBs is complete for the equivalent LTDBs, with data fibres fully connected between the LTDBs and LATOMEs for all of the ATCA crates set up in the ATLAS service cavern. 


\section{Main Readout Path Validation}

Although the main readout path is unchanged by the Phase-I upgrade, much of the contributing electronics has undergone refurbishment. Signals pass through new baseplanes to FEBs whose LSBs have been exchanged, and some of these FEBs have been returned to different positions within the HFECs, or to different HFECs entirely. Checking data from the main readout path is therefore an important first step in confirming that the system is performing as it was before any disturbances due to installation. Basic low-level checks such as connectivity scans are used to ensure that all FEBs and channels are connected properly, while full calibration sets with comparisons to reference runs from the end of Run- 2 provide a detailed check that the pulses read from the detector look the same as before, confirming that the electronics are stable. Three different types of calibration runs, namely, pedestal, delay and ramp runs, are used in order to calibrate the system and validate the readout electronics. Pedestal runs involve reading the detector output with no input signal, providing pedestal information from the average read out signal (ADC), noise from the RMS and autocorrelation from the timing correlation of the samples. Delay runs consist of injecting pulses with a single input signal current (DAC) with an increasing time shift in steps of $1.04 \mathrm{~ns}$ in order to reconstruct the full pulse shape. Ramp runs, where signals of increasing DAC are injected, provide the gain slope, which is extracted from a fit of the DAC versus ADC curve. Figure 6 shows, as an example, the comparison between pedestal RMS values measured before and after the installation process for the middle layer of EMBA. The data are in clear agreement, confirming that the system is stable and the hardware upgrades have not impacted this readout path. Such stability has been seen for all calibration run types in all of the refurbished crates.

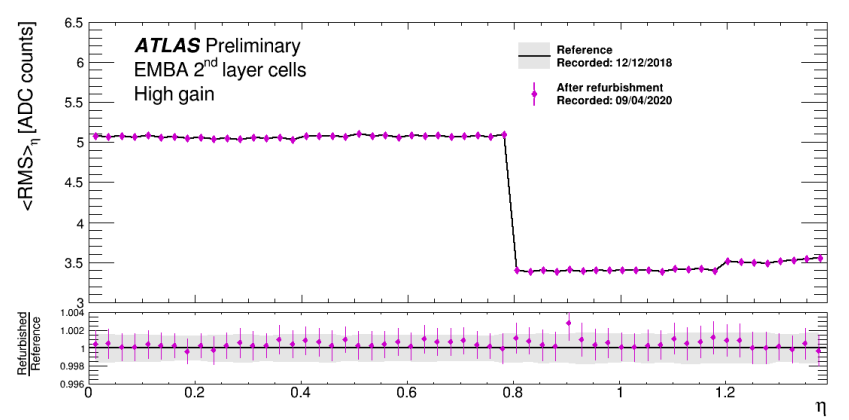

Figure 6: The mean value of the RMS of the pedestal for a given $\eta$ range measured during a reference run at the end of Run-2 (black line) compared to values measured after the front-end refurbishment (purple dots) during LS2 [10]. The data shown is only taken from the second layer of EMBA. The displayed uncertainties are computed as $\sigma_{r m s, c e l l s} / \sqrt{N_{\text {cells }}}$, where $\sigma_{r m s, \text { cells }}$ is the RMS of the RMS values over the cells in the given $\eta$ range and $N_{\text {cells }}$ is the number of cells in the given $\eta$ range. The step at $\eta=0.8$ can be attributed to a change of the electrode geometrical structure in this region.

\section{Legacy Trigger Path Validation}

As described in section III, the analogue legacy sums for some layers will be routed through the LTDBs before reaching the TT builders in the upgraded electronics scheme. This increased path through the system will introduce a delay in the L1 system for reception of the signal between different layers, which must be measured and accounted for in the trigger decision. Measuring these delays is an important step in validating the new hardware, since the introduced timing difference must reflect the expected performance of the system. Gain and timing scans performed in collaboration with the L1 trigger group are taken upon the refurbishment of each crate in order to check connectivity and measure this delay between the sums from different layers. Figure 7 shows the delays obtained from these timing scans for each layer in EMBA. A timing difference of up to $10 \mathrm{~ns}$ is observed between some layers, which is consistent with the expected change due to the increased path through the LTDB.

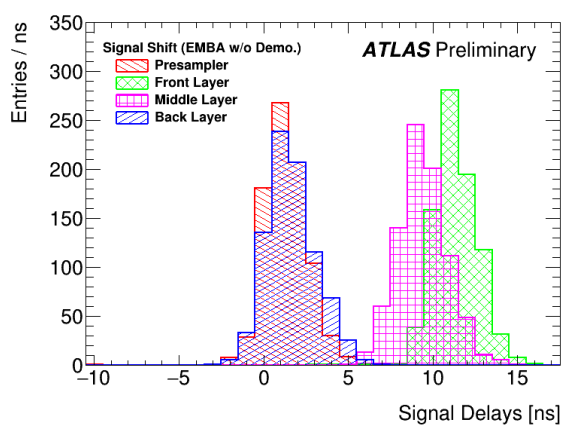

Figure 7: The signal delays introduced by the Phase-I front-end electronics for L1Calo trigger signals, collected from all trigger towers in EMBA [10]. Signals from the region $0<\eta<1.5$ and $1.8<\eta<2.2$ are excluded, since these were covered by Phase-I test electronics which were installed during Run-2.

\section{Digital Trigger Path Validation}

In order to validate the full digital trigger front-end and back-end path, the different calibration runs, as described in section V, are used, with ADCs for full SCs read from the LATOME output, as opposed to the individual LAr cell data from the main readout path. This validation requires the full chain of the Phase-I system, starting with configuration of the LTDBs and LDPS to take data. Special calibration patterns must also be developed, in order to pulse all of the cells in each tested SC. Overnight pedestal or pulsed runs are taken in order to monitor the stability of the system. These runs are also used to extract information such as the pedestal value and peak position for the pulses from each LATOME, which is obtained using data processing software which is automatically applied to the raw data following each run. Full pedestal, delay and ramp calibration sets are taken for each fully refurbished and upgraded crate. Figure 8 shows examples of processed calibration data from a gain and ramp run for a SC in EMBA. Here we see a pulse shape from the delay run, which has the expected bipolar 
form, and an ADC versus DAC curve from the ramp run. In the latter plot, the ADC increases linearly with deposited transverse energy up to around $800 \mathrm{GeV}$, where saturation of the SC pulse occurs. Such saturation is expected to occur in some channels for very high energies. Unlike the main readout, no reference data exists for this newly installed system, therefore validation of the digital trigger requires checking that the calibration data behaves as expected based on the predicted performance. In many cases, select variables from the processed data (such as pulse position or gain slope) are compared to expected thresholds, which are determined through an understanding of the expected SC output. From these runs, calibration constants used for energy reconstruction are obtained, and the offline $E_{T}$ can be compared to what is computed by the LATOME. For the LATOMEs tested so far, this $E_{T}$ is found to be in good agreement.
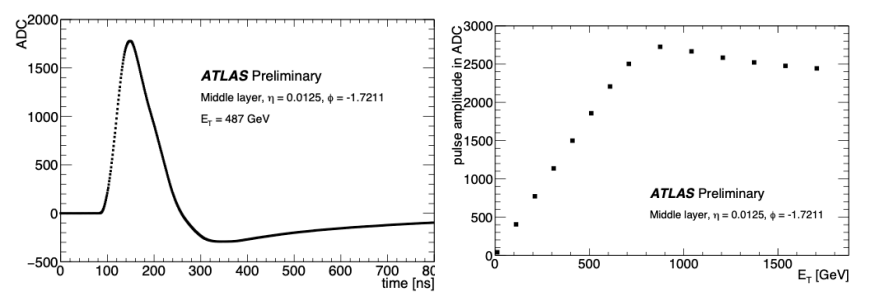

Figure 8: Calibration data read from the Phase-I trigger path [11]. The plot on the left shows the measured pulse shape of a SC in EMBA, obtained from delay run with an injected charge of DAC $=4400$ (corresponding to a transverse energy of $487 \mathrm{GeV}$ ). The plot on the right shows the measured amplitude (ADC) of calibration pulses as a function of injected charge (DAC) from a ramp run for this same SC.

\section{Conclusion}

In this contribution, an overview of the installation and commissioning of the ATLAS LAr Calorimeter Phase-I upgrade has been presented. Excellent progress has been made in the installation, despite additional challenges brought on by recent global events, with almost the full front-end and back-end electronics installed, pending access to the remaining sections of the detector. The commissioning of crates involves checks of the main readout, legacy trigger and digital trigger path. The main readout checks have shown that the system is stable, with no deviations from the performance seen at the end of Run-2, and the legacy trigger checks show the expected timing shifts introduced by LTDBs. Connectivity and mapping checks, as well as full calibration sets are being used to check newly connected Phase-I hardware, with automatic processing and comparisons to newly defined thresholds used to validate each crate. These tools will be used to sign-off all of the upgraded HFECs and to prepare for processing real data in upcoming pilot runs and Run-3, which starts in March of 2022.

\section{Acknowledgments}

I would like to thank the organisers of ANIMMA 2021, for inviting me to present these results and for organising a rich programme of diverse content, even during this difficult time. I would also like to thank my colleagues in the ATLAS Liquid Argon Calorimeter group for affording me the opportunity to present this content on their behalf.

\section{References}

[1] ATLAS Collaboration, "The ATLAS Experiment at the CERN Large Hadron Collider, "IINST vol. 3, S08003, 2008. doi: 10.1088/1748-0221/3/08/S08003.

[2] P. Krieger, "The atlas liquid argon calorimeter: Construction, integration, commissioning and performance from selected particle beam test results," in IEEE Nuclear Science Symposium Conference Record, 200.5 vol 2, 2005, pp. 1029-1033. doi: 10.1109/NSSMIC.2005. 1596428 .

[3] ATLAS Collaboration, "ATLAS Liquid Argon Calorimeter: Technical Design Report," ATLAS-TDR2: CFRN-LHCC-96-041, 1996. [Online]. Available: https://cds.cern.ch/record/331061.

[4] ATLAS Collaboration, "Drift Time Measurement in the ATLAS Liquid Argon Electromagnetic Calorimeter using Cosmic Munns," Fur Phys . I C, vol 70 p. 755, 2010. doi: 10.1140/epjc/s10052-010-1403-6. arXiv: 1002.4189 [hep-ex].

[5] N. J. Buchanan et al., "ATLAS liquid argon calorimeter front end electronics," Journal of Instrumentation, vol. 3, nn. 09, P09003-P09003, Sep. 2008. doi: 10 1088/ $1748-0221 / 3 / 0.9 /$ p09003. [Online]. Available: https : //doi.org/10.1088/1748-0221/3/09/p09003.

[6] ATLAS Collaboration, "ATLAS Level-1 Trigger: Technical Design Report," ATLAS-TDR-12; CFRN-I.HCC98-014, 1998. [Online]. Available: https://cds.cern.ch/ record/381429.

[7] ATLAS Collaboration, "ATLAS Liquid Argon Calorimeter Phase-I Upgrade: Technical Design Report," ATLAS-TDR-022. CF.RN-L.HCC-2013-017 2013 Online]. Available: https://cds.cern.ch/record/ 1602230.

[8] ATLAS Collaboration, "ATLAS TDAQ System PhaseI Upgrade: Technical Design Report," ATLAS-TDR023: CFRN-LHCC-2013-018, 2013. [Online]. Available: https://cds.cern.ch/record/1602235.

[9] CFRN-IPMC: Technical Overview [Online]. Available: https://cern-ipmc.web.cern.ch/doc.

[10] Level-1 Calorimeter Trigger Public Results. [Online]. Available: htt.ns: / / twiki cern ch / twiki / bin / view / AtlasPublic/L1CaloTriggerPublicResults.

[11] LAr Calorimeter LS2 Public Results. [Online]. Available: https : / / twiki cern . ch / twiki / bin / view / AtlasPublic/LArCaloPublicResultsLS2. 